\title{
Research on Construction Method of Operational Reliability Control Model for Space Manipulator Based on Particle Filter
}

\author{
Xin Gao, ${ }^{1}$ Yifan Wang, ${ }^{1}$ Hanxu Sun, ${ }^{1}$ Qingxuan Jia, ${ }^{1}$ Xiaojian Yang, \\ Mingtao Du, ${ }^{1}$ and Jingyu Huang ${ }^{1}$ \\ ${ }^{1}$ School of Automation, Beijing University of Posts and Telecommunications, No. 10, Xitucheng Road, Haidian District, \\ Beijing 100876, China \\ ${ }^{2}$ School of Automation, Beihang University, No. 37, Xueyuan Road, Haidian District, Beijing 100191, China
}

Correspondence should be addressed to Xin Gao; xlhhh74@bupt.edu.cn

Received 28 August 2015; Accepted 18 October 2015

Academic Editor: Panos Liatsis

Copyright (C) 2015 Xin Gao et al. This is an open access article distributed under the Creative Commons Attribution License, which permits unrestricted use, distribution, and reproduction in any medium, provided the original work is properly cited.

\begin{abstract}
The operational reliability of the space manipulator is closely related to the control method. However the existing control methods seldom consider the operational reliability from the system level. A method to construct the operational reliability system control model based on particle filter for the space manipulator is presented in this paper. Firstly, the definition of operational reliability and the degree of operational reliability are given and the state space equations of the control system are established as well. Secondly, based on the particle filter algorithm, a method to estimate the distribution of the end position error and calculate the degree of operational reliability with any form of noise distribution in real time is established. Furthermore, a performance model based on quality loss theory is built and a performance function is obtained to evaluate the quality of the control process. The adjustment value of the end position of the space manipulator can be calculated by using the performance function. Finally, a large number of simulation results show that the control method proposed in this paper can improve the task success rate effectively compared to the simulation results using traditional control methods and control methods based on Bayesian estimation.
\end{abstract}

\section{Introduction}

Space manipulators can assist or replace astronauts to finish complex and dangerous space tasks $[1,2]$. With the development of space technologies, space application tasks are becoming increasingly diverse, and the structure of space manipulators is also becoming more and more complicated [3-5]. Meanwhile, due to its harsh working environment and difficulty to repair and maintain, how to maintain a high reliability of space manipulator is a research hotspot and difficult point of space technology $[6,7]$.

The reliability of space manipulator includes the inherent reliability and the operational reliability [8], and the inherent reliability is given during its process of design and manufacture, relevant to various structural parameters of the manipulator and performance parameters of components [9]; the operational reliability is a character to maintain the performance ability shown in the actual use process of a product, which considers not only the influence factors of inherent reliability but also those which impacts product installation, operation, and repair support, and so forth [10, $11]$.

Currently, researches on reliability of space manipulator mainly focus on the inherent reliability, and related research achievements mainly include evaluating the reliability of a manipulator with a certain method, reliability design of manipulators, and fault-tolerant techniques of manipulators [12-18]. Because the space manipulator is a kind of controllable space mechanism, its operational reliability is closely related to its control method. Currently, the mainstream control methods for space manipulator, such as task planning method, path planning method, robust control method, and neural network method, take into account many factors, but the operational reliability of a manipulator is not considered from the system level [19-27]. This may cause a low reliability of the system and cannot guarantee a manipulator 
to complete the task sustainably and reliably. Therefore, in order to further improve the operational reliability of space manipulator, system control method comprehensive multiple factors of operational reliability should be considered, building control methods and adjustment strategies to maintain its operational reliability from the system level. When the space manipulator performs specified tasks, through the adjustment of control strategies and self-adaptive changes, the ability of completing a task successfully with specified control method is maintained to improve the operational reliability of the system under the condition of constant inherent reliability.

A method to construct the operational reliability system control model for space manipulator based on the particle filter is proposed in this paper. Combining reliability theory with control characteristics of space manipulator, based on the given definition of the operational reliability and the degree of operational reliability for space manipulator, the state space equations of the control system are established. Combined with the principle of particle filter algorithm and features of the control system, the estimation of the terminal position error distribution and real time calculation of the operational reliability for space manipulator under any distribution of observed noise are realized. Control process performance model based on the principle of quality loss is constructed, and the control performance function is obtained, according to which the calculation of the terminal position adjustment amount for space manipulator is achieved. In this method, the operational reliability of space manipulator is calculated at the same time as the execution of specified tasks, and the control of manipulator is adjusted according to the degree of operational reliability; thus operational reliability and working efficiency of space manipulator are improved. Simulation results show that, compared with traditional control method and that based on Bayesian estimation, successful rate of tasks is increased significantly and the operational reliability of manipulator is improved effectively by the proposed control method.

\section{Construction of Operational Reliability Control Model for Space Manipulator}

\subsection{Description of Operational Reliability for Space Manip-} ulator. The operational reliability of space manipulator is the reliability shown in the process of its actual use, and it depends on its control method because it is a kind of controllable device. In this paper, the operational reliability of space manipulator is defined as the ability to complete the task successfully with specified control method when it performs the specified task, which is quantified by the degree of the operational reliability, and specifically reflected as the probability of completing the task for space manipulator according to specified requirements [28].

According to the control characteristics of space manipulator, its work consists of a series of tasks, which is denoted by $T$,

$$
T=\left\{T_{i} \mid i=1,2, \ldots, n\right\}
$$

For any given task $T_{i}, m$ factors which determine the success or failure of the task are denoted by $I_{i}$,

$$
I_{i}=\left\{I_{i j} \mid j=1,2, \ldots, m\right\} .
$$

For each $I_{i j}$ of any given task $T_{i}$, it has a prescribed accuracy requirement, which is denoted by the following interval:

$$
E_{i j}=\left[\lambda_{i j}-\varepsilon_{i j}, \lambda_{i j}+\varepsilon_{i j}\right]
$$

where $\lambda_{i j}$ is the expectation of $I_{i j}$ and $\varepsilon_{i j} \in[0,+\infty)$ presents the error allowance of $I_{i j}$. So if $I_{i j} \in E_{i j}(i=1,2, \ldots, n, j=$ $1,2, \ldots, m)$ when $T_{i}$ is finished, task $T_{i}$ is considered executed successfully.

After the task $T_{i}$ is completed using a specified control method, a nonnegative random variable $X_{i j}$ is used to describe the value of $I_{i j}$, the distribution function of which is described as

$$
F_{i j}\left(\lambda_{i j}\right)=P\left\{X_{i j}<\lambda_{i j}\right\}, \quad j=1,2, \ldots, m .
$$

On this basis, the probability of $X_{i j}$ meeting accuracy requirements of task $T_{i}$ can be obtained as follows:

$$
R\left(E_{i j}\right)=P\left\{\bigcap_{j=1}^{m} X_{i j} \in E_{i j}\right\},
$$

where $R\left(E_{i j}\right)$ is the operational reliability degree of space manipulator. From formula (5), the operational reliability degree of a manipulator is the probability that each factor meets specified accuracy requirement when it performs a task. Therefore, the operational reliability of a space manipulator can also be defined as the probability of successful completing of specified task when using specified control method to control the manipulator. So $I_{i j}$ can also be called the reliability influence factor, and it can have different definitions such as terminal position and terminal force, according to different tasks.

\subsection{Basic Components of the Operational Reliability Control} System for the Manipulator. As is shown in Figure 1, the operational reliability control system for the space manipulator is constructed based on its general control system, and the real time calculation of the operational reliability degree is a feedback unit of the control system. The basic working principle of the control system is as follows: the error value between the terminal position and the specified position of the space manipulator is measured after the movement control unit by the System Condition Detection Module, according to which the operational reliability degree of the space manipulator system is estimated and then the adjustment amount of its control variables is calculated. So the control variables can be fine-tuned to reduce the error and improve the system reliability.

The control system schematic diagram of the space manipulator adding the operational reliability control loop is shown in Figure 1. The overall working process of the control system is as follows: first, the task planning module divides 


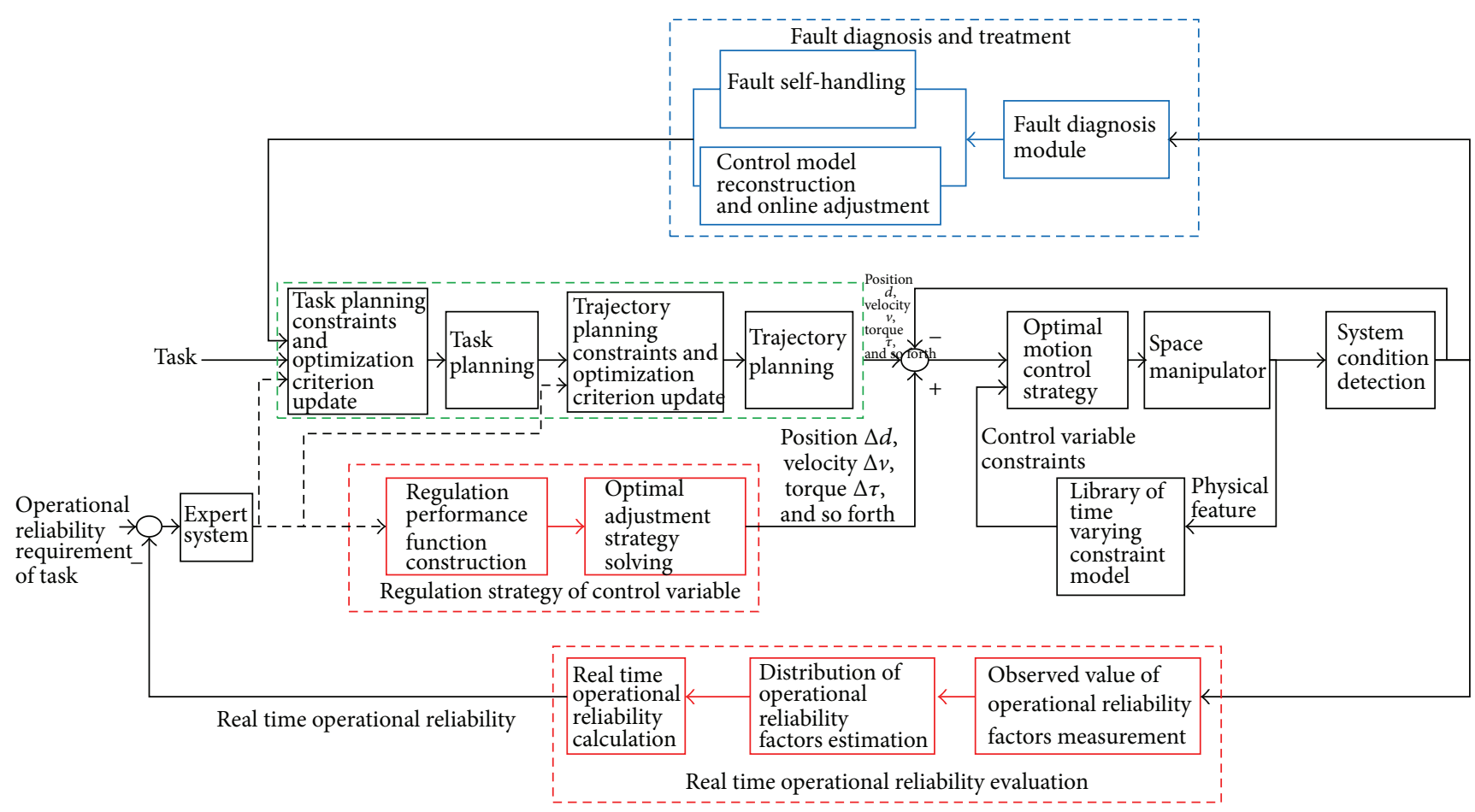

FIGURE 1: The structure diagram of the operational reliability control system for space manipulator.

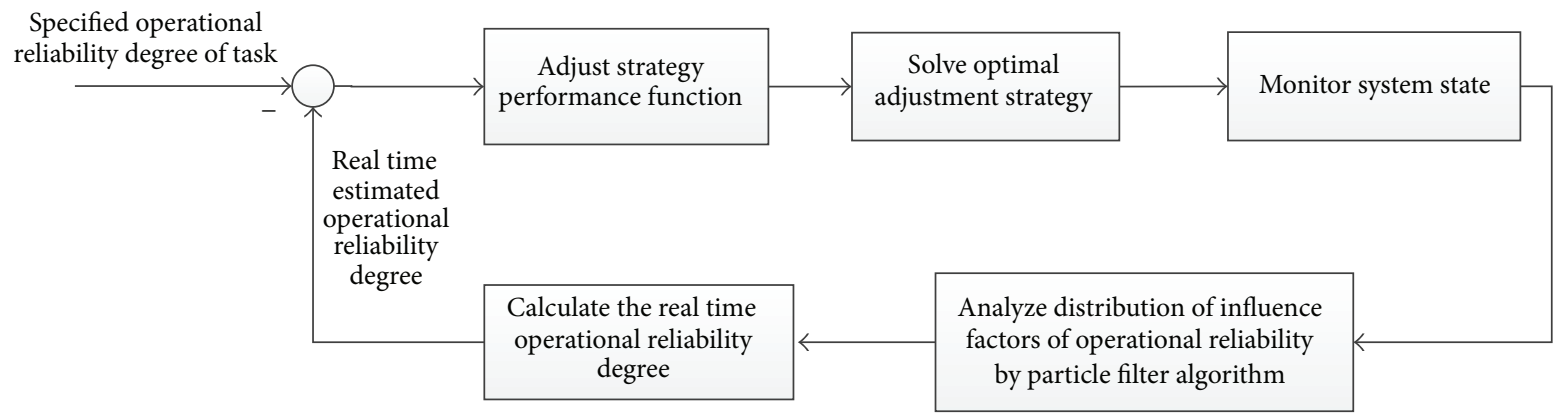

FIGURE 2: Independent structure of operational reliability control system for space manipulator.

the specified task into a series of subtasks and trajectory waypoints consistent with the constraints, and subtasks will be input to the path planning module to obtain the optimal path. Thus, various control parameters of the manipulator are given, such as position, velocity, and force, which are input to motion control module to solve the optimal control strategy and then to drive the actuator to complete the task. There are three feedback loops in the control system. The first one is used within the motion control module to monitor movements of the manipulator in real time; the second one is applied to detect and process faults of space manipulator in real time and adjust the control strategy according to the faults; the last one is the control circuit of the operational reliability, which is used to detect the operational reliability of the space manipulator and amend the control parameters of space manipulator according to the operational reliability.

A less detailed representation for the operational reliability control system in Figure 1 is shown in Figure 2.
The working process of the operational reliability system for space manipulator described in Figure 2 is as follows: observed values of influence factors of operational reliability are first obtained by system state monitoring module, and the particle filter algorithm is used to analyse the real distribution of the influence factors to calculate the operational reliability degree, which is compared with the specified reliability to solve the optimal adjustment strategy and adjust the control amount of space manipulator accordingly. Among them, the real time calculation of the operational reliability degree and the solution of optimal adjustment strategy are two key aspects of the system.

\subsection{State Space Model of the Operational Reliability Control} System for Manipulator. General operating mode of space manipulator in orbit mainly includes no-load operation, load operation, and a mixed one. In this paper, the proposed operational reliability control system for space manipulator 
is assumed that it is working under no-load running state. Under this state, whether the implementation results meet requirement only depends on its terminal position. Assume the error of terminal position is $\theta$ and the following discrete state space equations are established to describe the control process based on the control system model:

$$
\begin{aligned}
& \theta_{i}=\theta_{i-1}+x_{i-1}, \\
& y_{i}=\theta_{i}+v_{i},
\end{aligned}
$$

$$
i=1,2, \ldots, N \text {, }
$$

where $\theta_{i}$ is the actual error of terminal position for the $i$ th control cycle; $y_{i}$ presents terminal position error for the $i$ th control cycle obtained by forward kinematics solution with noise; $x_{i}$ is the adjustment amount of control variable for the $i$ th control cycle and $v_{i}$ is the observed noise error, which can obey any distribution.

The above state space model requires that a control task of the control system for the space manipulator has two or more control cycles. The initial distribution of terminal position error for space manipulator is obtained in the first control cycle, and then it is amended according to observations of terminal position error in later control cycles. In each control cycle, operational reliability of space manipulator is calculated based on the obtained distribution of terminal position error, which is used to calculate adjustment amount of the control variable for adjusting, so the error is gradually reduced and operational reliability of space manipulator is gradually improved.

Expression of the operational reliability for space manipulator in this paper can be obtained by the general formula of the operational reliability in formula (5) and can be written as

$$
R=P\left\{\theta_{i 1} \leq \theta \leq \theta_{i 2}\right\} .
$$

\section{Real Time Control of the Operational Reliability Control System for Manipulator}

When performing a given task, the space manipulator needs to arrive at the preset space position at the preset time. However, due to the impact of the internal joints gear clearance and sensor noise and, and so forth, after performing a certain task within a number of control cycles, there is a certain error between the actual spatial position and the expected spatial position. If not corrected, it is possible to lead to a bigger and bigger error between the actual execution results with the expected implementation results and finally reduce the operational reliability of the space manipulator.

Because there may be difference on the internal structure or the environment, the real position error distribution of the space manipulator may not be considered as the simple Gaussian distribution [28], and it may obey all sorts of distribution which is difficult to be described in some analytical methods. At this moment it may lead to great deviation calculation results compared to the actual problem when using the classical Bayesian estimation method. So the estimation of the terminal position error distribution and real time calculation of the operational reliability degree for space manipulator under any distribution of observed noise are realized using the particle filter algorithm in this paper.

3.1. Using Particle Filter Algorithm to Calculate the Operational Reliability Degree. The basic idea of particle filter algorithm is that two sets of particles and particle weights are used to describe the target states, where particles represent some samples of target states and particle weights represent the degree of similarity between particles and target states [29]. In the changing process of the target parameters, the target states can be tracked and predicted through continuous revision of particles and their weights.

Combined with state space model and the principle of particle filter algorithm, two sets of $\left\{z_{i}^{j}, j=1, \ldots, N_{s}\right\}$ and $\left\{\omega_{i}^{j}, j=1, \ldots, N_{s}\right\}$ are used to describe the distribution of terminal position error $\theta_{i}$, where $\left\{z_{i}^{j}, j=1, \ldots, N_{s}\right\}$ is a group of sample data (namely, particles) in the same form of $\theta_{i}$ and $N_{s}$ represents the number of particles; $\left\{\omega_{i}^{j}, j=1\right.$, $\left.\ldots, N_{s}\right\}$ is the corresponding weights of particles, representing the relevance degree of particles $z_{i}^{j}$ and $\theta_{i}$ and $\sum_{j=1}^{N_{s}} \omega_{i}^{j}=$ 1.

In addition, the following normal distribution model is used as a priori probability distribution of $\theta_{i}$ :

$$
\theta_{0} \sim N\left(\mu_{0}, \Lambda_{0}\right),
$$

where $\theta_{0}$ is the initial value of the terminal position actual error for space manipulator, with $\mu_{0}$ and $\Lambda_{0}$ being the expectation and variance of a priori probability distribution for $\theta_{0}$, respectively.

Then the particle filter is conducted based on state space equation of the system and a priori distribution, and the main steps are as follows.

(1) Initialization: for the first control cycle of task, the number of $N_{s}$ random quantities is generated as the initial particle set $\left\{z_{0}^{j}, j=1, \ldots, N_{s}\right\}$ based on a priori probability distribution of $\theta_{0}$, with their corresponding weights set as $\left\{\omega_{0}^{j}=1 / N_{s}, j=1, \ldots, N_{s}\right\}$. For the second or later control cycles, the particle set is obtained by resampling according to the particle $\left\{z_{i-1}^{j}, j=1, \ldots, N_{s}\right\}$ and its weight $\left\{\omega_{i-1}^{j}, j=\right.$ $\left.1, \ldots, N_{s}\right\}$ of the previous control cycle and can be described as

$$
\begin{aligned}
z_{i}^{j} & =z_{i-1}^{k}+x_{i-1}, \\
\sum_{j=1}^{k} \omega_{i}^{j} & \geq e_{i}^{j}, \\
\sum_{j=1}^{k-1} \omega_{i}^{j} & \leq e_{i}^{j},
\end{aligned}
$$

where $i$ is an integer greater than or equal to $1, z_{i-1}^{j}$ is the particle for the $(i-1)$ th control period, $x_{i-1}$ is the adjustment 
amount of the end position error of the space manipulator for the $(i-1)$ th control period, and $e_{i}^{j}$ is a random number satisfying the uniform distribution between 0 and 1 .

(2) The value of weight is updated according to the importance function using a priori distribution model, which is given as follows:

$$
\omega_{i}^{j} \propto \omega_{i-1}^{j} p\left(y_{i} \mid z_{i}^{j}\right)
$$

Due to using of the method of resampling, the weight value of the particle is $\omega_{i-1}^{j}=1 / N_{s}$, so there is

$$
\omega_{i}^{j} \propto p\left(y_{i} \mid z_{i}^{j}\right) .
$$

$p\left(y_{i} \mid z_{i}^{j}\right)$ is related to the noise distribution, and the noise $v_{i}$ is set to obey the distribution $\Phi$ with a probability density function of $h(\rho)$, where $\rho$ is a variable. So the calculation function of $\omega_{i}^{j}$ is

$$
H\left(z_{i}^{j}\right)=h\left(y_{i}-z_{i}^{j}\right)
$$

That is to say, $\omega_{i}^{j}=h\left(y_{i}-z_{i}^{j}\right)$.

(3) The weight value $\omega_{i}^{j}$ is normalized; namely, it is set that, $\sum_{j=1}^{N_{s}} \omega_{i}^{j}=1$.

Based on the above methods, the probability distribution function $f\left(\theta_{i} \mid y_{i}\right)$ of the terminal position error for space manipulator is obtained as

$$
f\left(\theta_{i} \mid y_{i}\right) \approx \sum_{j=1}^{N_{s}} \omega_{i}^{j} \delta\left(\theta_{i}-z_{i}^{j}\right),
$$

where the formula of $\delta(x)$ with $x$ as its variable is as follows:

$$
\delta(x)= \begin{cases}1, & x \neq 0 \\ 0, & x=0 .\end{cases}
$$

Then, according to $f\left(\theta_{i} \mid y_{i}\right)$, the operational reliability degree of space manipulator is obtained by using the following formula:

$$
\begin{aligned}
P\left\{\theta_{i 1} \leq \theta_{i} \leq \theta_{i 2}\right\} & =\int_{\theta_{i 1}}^{\theta_{i 2}} f\left(\theta_{i} \mid y_{i}\right) d \theta_{i} \\
& =\sum_{j=1}^{N_{s}} \begin{cases}\omega_{i}^{j}, & \theta_{i 1} \leq z_{i}^{j} \leq \theta_{i 2} \\
0, & \text { otherwise }\end{cases}
\end{aligned}
$$

where $\theta_{i 1} \leq \theta_{i} \leq \theta_{i 2}$ is the range of the preset precision, with $\theta_{i 1}$ and $\theta_{i 2}$ being lower and upper limit value of precision range, respectively, and satisfying $\theta_{i 2} \geq \theta_{i 1}$.

3.2. Real Time Adjustment Strategy of Control Variables for the Operational Reliability Control System. For the ith control cycle of control link, the following performance function is established to evaluate the control strategy [30],

$$
L_{i}=E\left[y_{i+1}^{T} y_{i+1}+c \delta\left(x_{i}\right)\right] \text {, }
$$

where $y_{i+1}$ is the observed value of the terminal position error of space manipulator for the $(i+1)$ th control cycle and $x_{i}$ is adjustment amount of control variable. The above formula can be considered as the function of $x_{i}$ when $\theta_{i}$ value is constant. Therefore, the best adjustment strategy in the control process is to calculate the optimal value of $x_{i}$, making the value of the above performance function as small as possible.

$L_{i}^{*}\left(\mu_{i}\right)$ and $L_{i}\left(\mu_{i}\right)$ are set as the minimal expectation loss for the $(i+1)$ th control cycle when the estimation value of the terminal position error for space manipulator is $\mu_{i}$ under the condition of adjustment and no adjustment, respectively. Adjustment amount $x_{i}$ is set as $x_{i}=k_{i} \mu_{i}$ to simplify the calculation, where $k_{i}$ is a one-dimensional variable.

From the definition of $L_{i}$, it can be obtained that

$$
L_{i}=\min \left\{L_{i}\left(\mu_{i}\right), L_{i}^{*}\left(\mu_{i}\right)\right\} .
$$

According to the definition of $L_{i}^{*}\left(\mu_{i}\right)$, it can be obtained that

$$
L_{i}^{*}\left(\mu_{i}\right)=\min _{x_{i}}\left\{E\left[y_{i+1}^{T} y_{i+1}+c \delta\left(x_{i}\right)\right]\right\} .
$$

Based on the state equations in formula (6), it can be obtained that

$$
\begin{aligned}
& L_{i}^{*}\left(\mu_{i}\right) \\
& \quad=\min _{x_{i}}\left\{E\left[\left(\theta_{i+1}+v_{i+1}\right)^{T}\left(\theta_{i+1}+v_{i+1}\right)+c \delta\left(x_{i}\right)\right]\right\} \\
& \quad=\min _{k_{i}}\left\{\left(1+k_{i}\right)^{2}\left\|\mu_{i}\right\|^{2}+\left(1+k_{i}\right)\left(\mu_{i}^{T} \alpha+\alpha^{T} \mu_{i}\right)\right. \\
& \left.\quad+\operatorname{tr}\left(\beta+\Lambda_{i}\right)+\|\alpha\|^{2}+c \delta\left(k_{i}\right)\right\} .
\end{aligned}
$$

That is to say,

$$
\begin{aligned}
& L_{i}^{*}\left(\mu_{i}\right)=\operatorname{tr}\left(\beta+\Lambda_{i}\right)+\|\alpha\|^{2}+\min _{k_{i}}\left\{\left(1+k_{i}\right)^{2}\left\|\mu_{i}\right\|^{2}\right. \\
& \left.\quad+\left(1+k_{i}\right)\left(\mu_{i}^{T} \alpha+\alpha^{T} \mu_{i}\right)+c \delta\left(k_{i}\right)\right\} .
\end{aligned}
$$
$\left.c \delta\left(k_{i}\right)\right\}$.

Make $R\left(\mu_{i}\right)=\min _{k_{i}}\left\{\left(1+k_{i}\right)^{2}\left\|\mu_{i}\right\|^{2}+\left(1+k_{i}\right)\left(\mu_{i}^{T} \alpha+\alpha^{T} \mu_{i}\right)+\right.$ Under the condition of $k_{i}=0$, it is known that

$$
R\left(\mu_{i}\right)=\left\|\mu_{i}\right\|^{2}+\mu_{i}^{T} \alpha+\alpha^{T} \mu_{i} .
$$

At this time, $L_{i}^{*}\left(\mu_{i}\right)=L_{i}\left(\mu_{i}\right)$ is obtained.

Under the condition of $k_{i} \neq 0$, there is the following formula which is equal to solve the minimum value of a quadratic function about $k_{i}$ :

$$
\begin{aligned}
& R\left(\mu_{i}\right)=\min _{k_{i}}\left\{\left(1+k_{i}\right)^{2}\left\|\mu_{i}\right\|^{2}\right. \\
& \left.\quad+\left(1+k_{i}\right)\left(\mu_{i}^{T} \alpha+\alpha^{T} \mu_{i}\right)+c\right\} .
\end{aligned}
$$

Due to the fact that $\left\|\mu_{i}\right\|^{2}>0$, the minimum value is obtained at the symmetry axis; that is,

$$
k_{i}=-1-\frac{\mu_{i}^{T} \alpha+\alpha^{T} \mu_{i}}{\left\|\mu_{i}\right\|^{2}},
$$


TABLE 1: The D-H parameters of the manipulator.

\begin{tabular}{lcccc}
\hline Link $i$ & $\alpha(\mathrm{rad})$ & $a(\mathrm{~mm})$ & $\theta(\mathrm{rad})$ & $d(\mathrm{~mm})$ \\
\hline 1 & 0 & 0 & 0 & 675 \\
2 & $-\pi / 2$ & 0 & $-\pi / 2$ & 110 \\
3 & $\pi / 2$ & 0 & 0 & 245 \\
4 & 0 & 135 & 0 & 0 \\
5 & 0 & 135 & $-\pi / 2$ & 0 \\
6 & $-\pi / 2$ & 0 & 0 & 310 \\
7 & $\pi / 2$ & 0 & 0 & 0 \\
8 & $-\pi / 2$ & 0 & 0 & 216 \\
\hline
\end{tabular}

where $\mu_{i}$ is the expectation of the probability distribution for the end position error of the space manipulator after the completion of the $i$ th control cycle and $\alpha$ is the noise expectation of control system, which can be obtained by the statistics of the noise samples and is a known amount.

So, the formula $L_{i}^{*}\left(\mu_{i}\right)=L_{i}\left(\left(1+k_{i}\right) \mu_{i}\right)+c$ is obtained at this time, where $c$ is the normalized adjustment costs; that is to say,

$$
L_{i}^{*}\left(\mu_{i}\right)=\min \left\{L_{i}\left(\mu_{i}\right), c+L_{i}\left(\left(1+k_{i}\right) \mu_{i}\right)\right\} .
$$

Therefore,

$$
\begin{aligned}
& \min \left\{L_{i}\left(\mu_{i}\right), L_{i}^{*}\left(\mu_{i}\right)\right\} \\
& =\min \left\{L_{i}\left(\mu_{i}\right), c+L_{i}\left(\left(1+k_{i}\right) \mu_{i}\right)\right\} .
\end{aligned}
$$

Meanwhile, the adjustment amount of the terminal position for space manipulator can be obtained as

$$
x_{i}\left(\mu_{i}\right)= \begin{cases}k_{i} \mu_{i}, & L_{i}\left(\mu_{i}\right) \geq c+L_{i}\left(\left(1+k_{i}\right) \mu_{i}\right) \\ 0, & L_{i}\left(\mu_{i}\right) \leq c+L_{i}\left(\left(1+k_{i}\right) \mu_{i}\right),\end{cases}
$$

where the formula of $\mu_{i}$ is obtained by particle filter algorithm and can be described as

$$
\mu_{i}=\sum_{j=1}^{N_{s}} \omega_{i}^{j} z_{i}^{j}
$$

\section{Simulation and Verification}

Based on the proposed control system model of the operational reliability and the corresponding control method, MATLAB software is used to establish a simulation system to verify its control effect. The object manipulator of study in this paper has 8 degrees of freedom. The manipulator's D-H coordinate system is shown in Figure 3. The D-H parameters are shown in Table 1.

The basic workflow of the simulation system is as follows: (1) initializing relevant parameters, such as the establishment of a collection of particles and their weights, the determination of the initial value of error, and so on, (2) estimating the distribution of the terminal position error for space manipulator by particle filter algorithm and calculating the degree of the operational reliability, and (3) determining

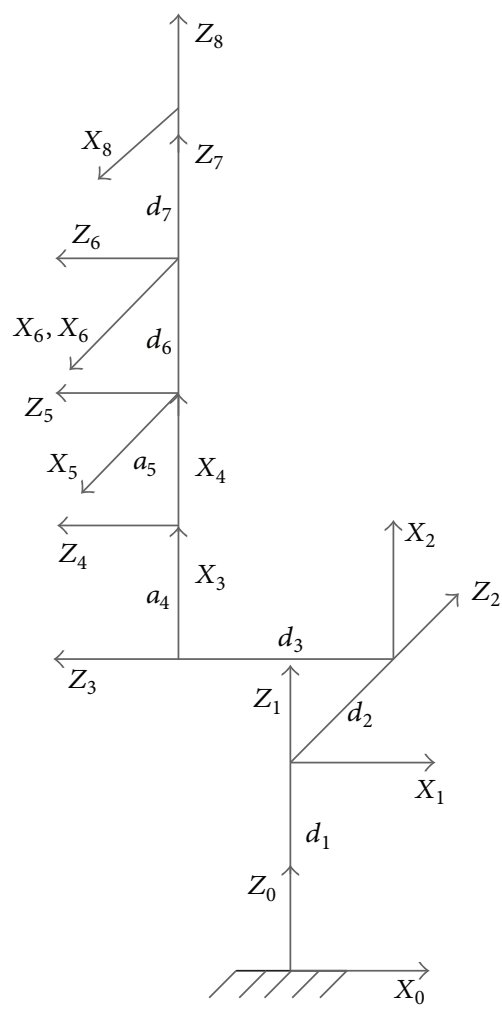

FIGURE 3: 8-DOF manipulator's D-H coordinate system.

the value of adjustment amount by the performance function, and so forth. A flowchart of the simulation system for a control cycle is shown in Figure 4.

4.1. The Basic Simulation. Based on the model structure of the operational reliability control system for space manipulator, a simulation system is established to verify its control effect, where the initial value of the terminal position error is $7 \mathrm{~mm}$ with an initial particle distribution $\theta_{0} \sim N(7,1)$ selected for particles, and assume the total number of the task control cycles is 9 with a specified upper and lower limit accuracy of $\pm 1 \mathrm{~mm}$ and a threshold reliability of 0.9 . The distribution curve of the observed noise used in this paper is shown in the solid blue line of Figure 5 .

The result shown in Figure 6 is obtained by a single simulation. As can be seen from Figure 6, the real value of the terminal position error for space manipulator in the control process gradually approaches 0 with no fluctuation, but the observed value has a large fluctuation due to the presence of noise. Compared with the observed value, the predicted value is relatively stable and gradually close to the real value. The degree of the operational reliability of space manipulator has a trend of continuous improving and gradual converging to 1 in the control process, which is shown by the change curve of that in Figure 7.

The 1000 times' simulation results are shown in Figure 8, where the horizontal axis is precision value and the vertical axis is times of execution result precision falling in the corresponding precision of the horizontal axis. A successful 


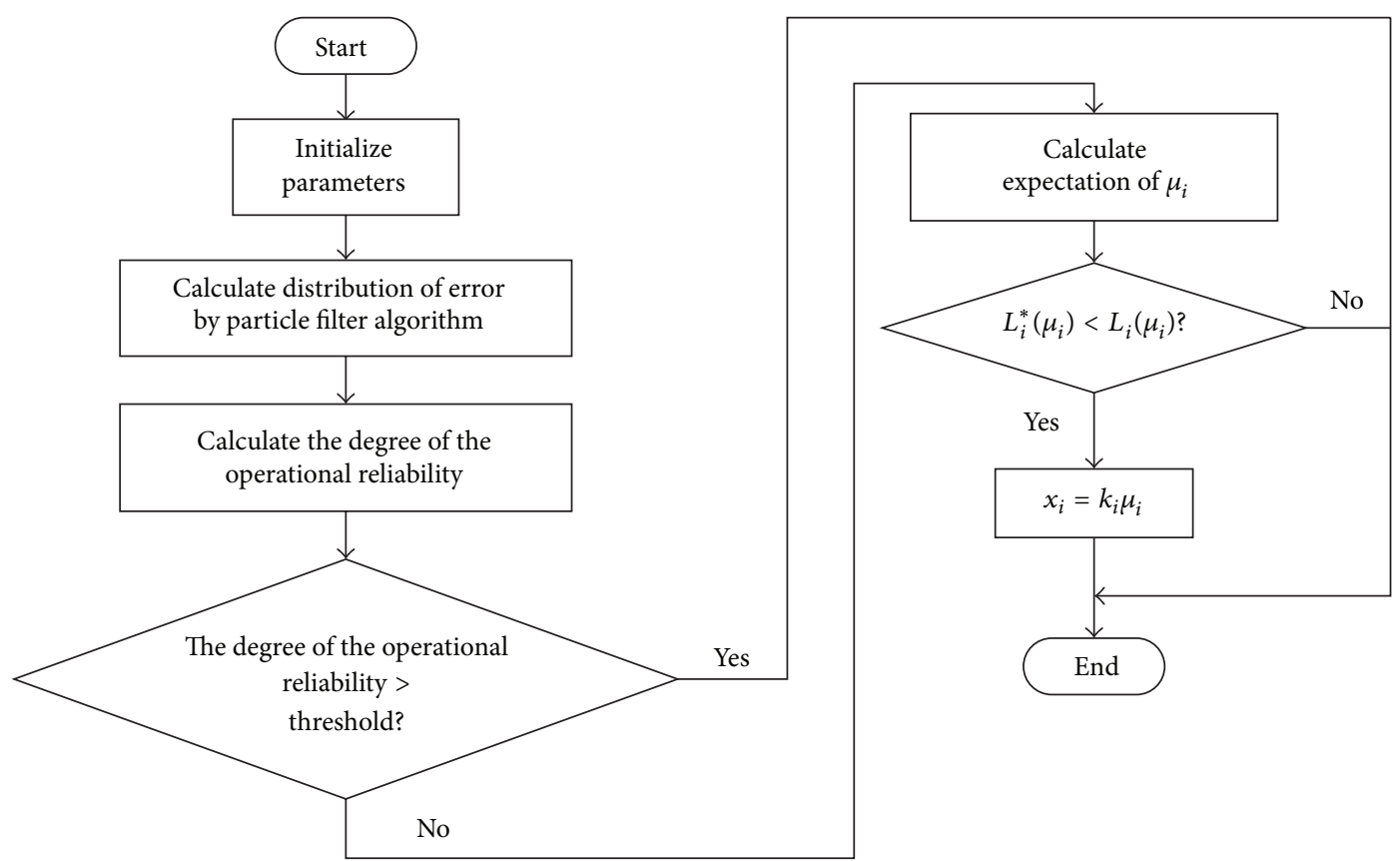

FIGURE 4: Flowchart of the simulation control system.

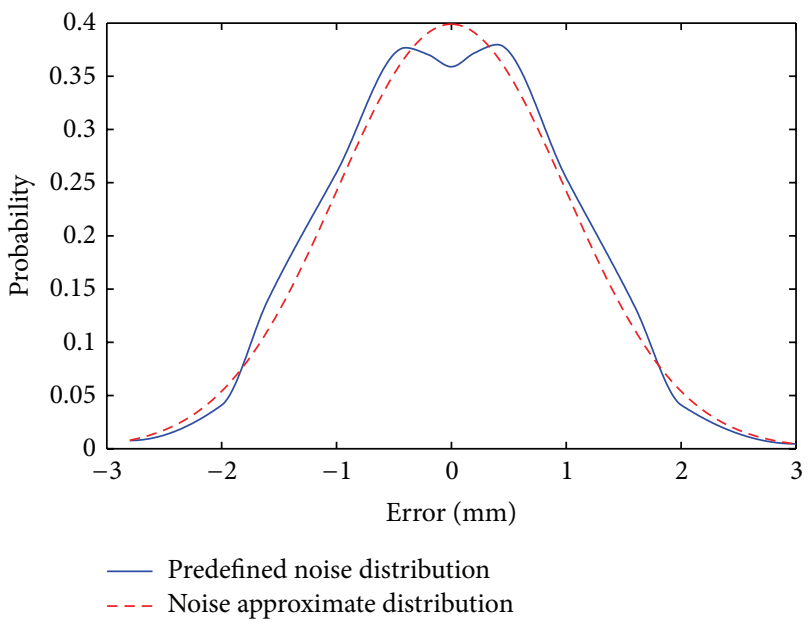

FIGURE 5: The distribution curve of observed noise.

rate of $87 \%$ is obtained on condition that a task is identified successfully when the final value of terminal position error for space manipulator in a control task is located within the stipulated range of $\pm 1 \mathrm{~mm}$, and the total loss of task calculated by performance function is 64855 in the control process.

\subsection{Contrast}

4.2.1. Simulation of Traditional Control Method. For the traditional control method, the actual value of terminal position error is directly adjusted by its observed value without calculating the operational reliability; namely,

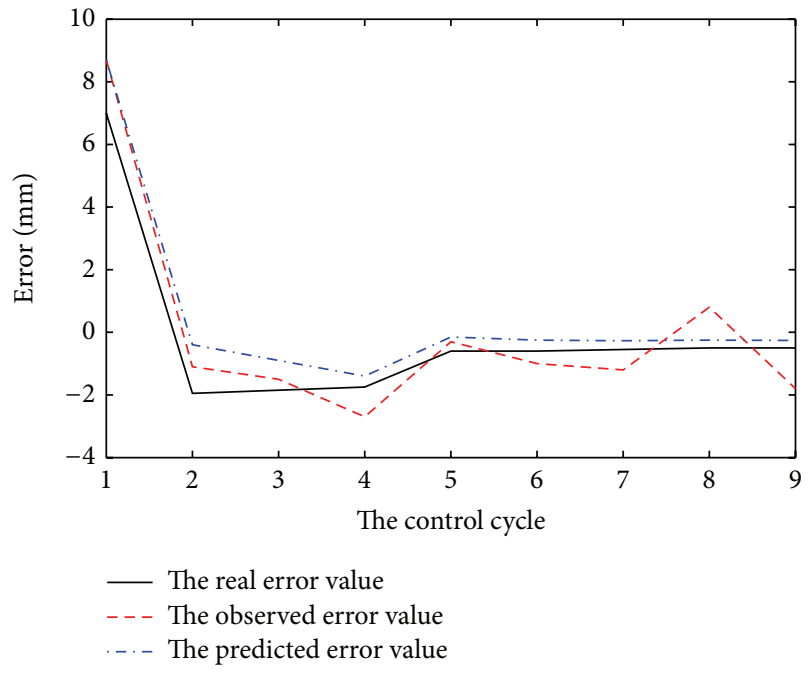

FIGURE 6: Distribution curve of error for a single simulation.

$x_{i}=-y_{i}$. Single and multiple simulation results, respectively, are as shown in Figures 9 and 10.

From the single simulation result shown in Figure 9, under the conditions without estimating the error of the terminal position and calculating the operational reliability, it can be seen that the real error of the terminal position for space manipulator decreases gradually and constantly fluctuates near 0 but cannot converge. The precision distribution curve for 1000 times is shown in Figure 10, where a calculated successful rate of task for the traditional control method is $60 \%$ and the total loss of task is 75336 . By contrast, 


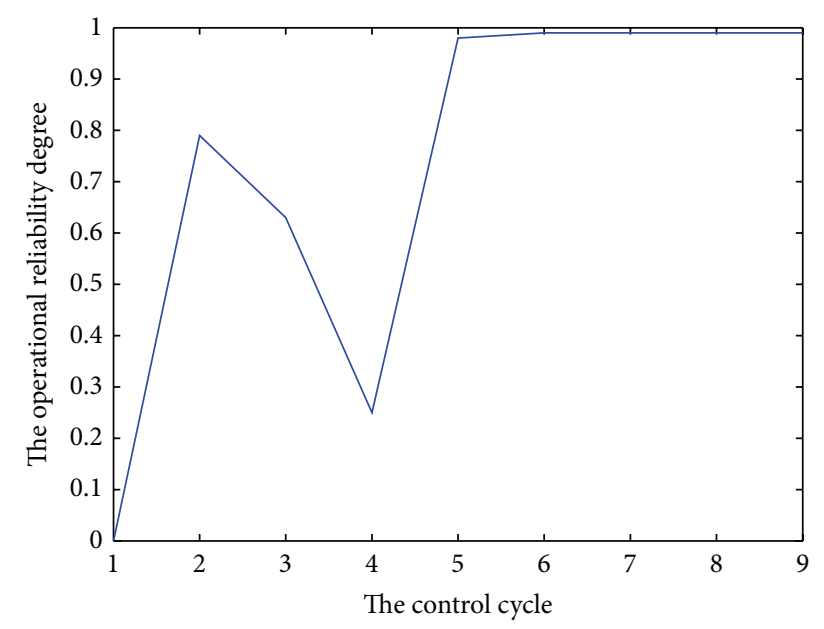

FIGURE 7: Curve of reliability for a single simulation.

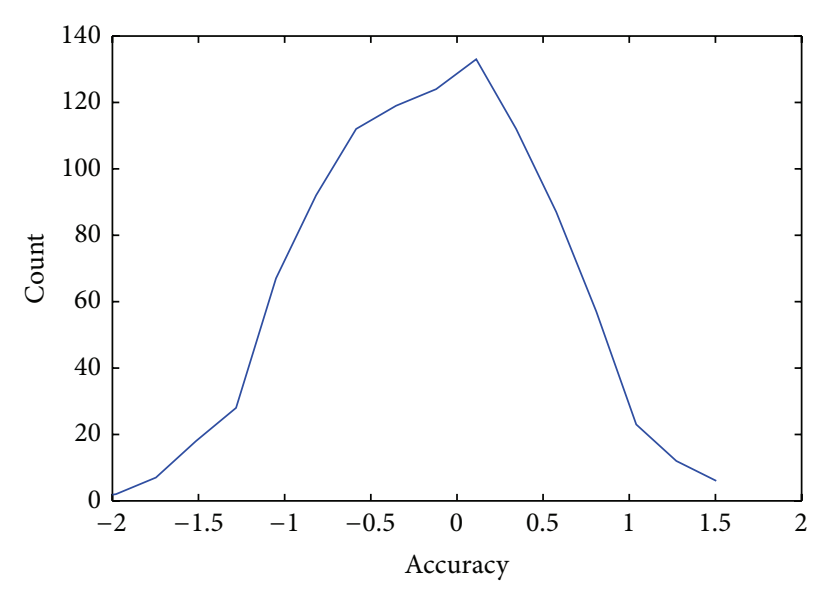

FIGURE 8: Distribution curve of precision for multiple simulation.

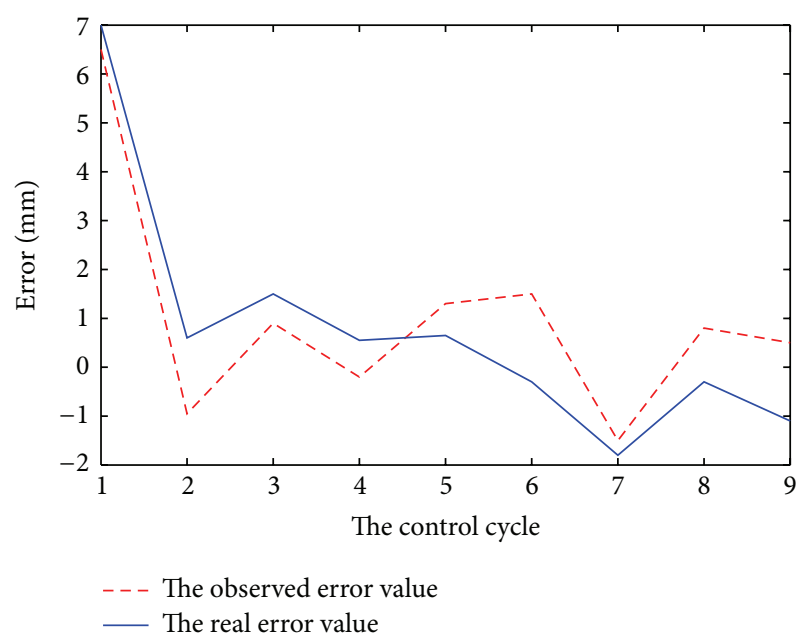

FIGURE 9: Error distribution curve of traditional control method for a single simulation.

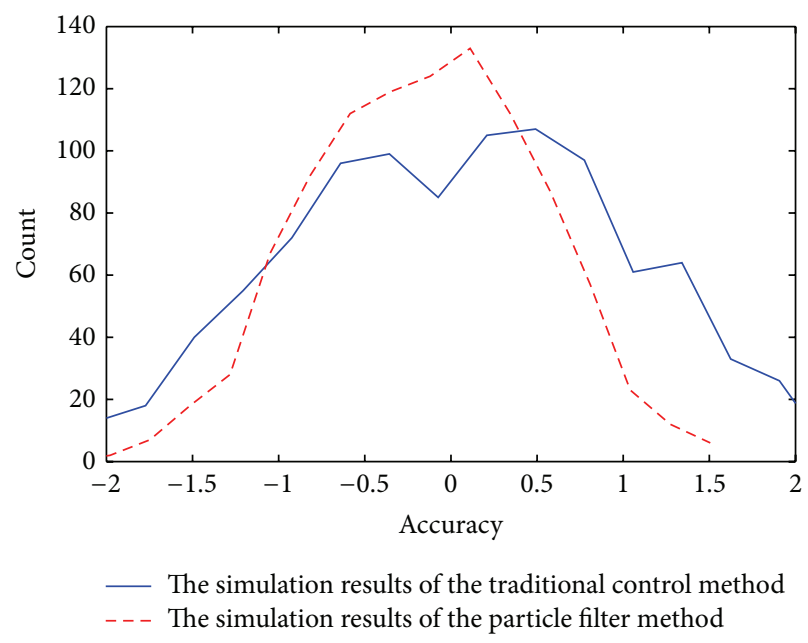

FIgURE 10: Precision distribution curve of traditional control method for multiple simulation.

the control effect of the presented method is better than that of the traditional method.

4.2.2. Simulation of Bayesian Estimation Method. Bayesian estimation theory is an estimation method based on the probability distribution [31], whose basic principle is that an unknown parameter $x$ is made as a random variable and its prior distribution $p(x)$ is introduced, which is amended according to the measured data and Bayesian theorem so as to obtain posterior probability density function of the parameter $x$. The distribution of the observed noise used in this paper needs to be treated approximately when Bayesian method is used to estimate that of the terminal position error. The approximate distribution curve of observed noise is shown as the dotted red line of normal distribution curve in Figure 11 with an expectation of 0 and a variance of 1 .

The principle of Bayesian estimation method is as follows [28].

First, a normal prior model is used as the prior probability distribution of $\theta_{i}$; that is, $\theta_{0} \sim N\left(\mu_{0}, \Lambda_{0}\right)$ and observed noise is set to obey normal distribution (namely, $v_{i} \sim N(0, \Sigma)$ ), where $\Sigma$ is an approximate variance.

Then, recursive posterior probability distribution of $\theta_{i}$ based on state space equations and Bayesian formula is as follows:

$$
\theta_{i} \mid y_{i} \sim N\left(\mu_{i}, \Lambda_{i}\right)
$$

where

$$
\begin{aligned}
\mu_{i} & =\frac{\Sigma^{-1} y_{i}+\Lambda_{i-1}^{-1}\left(\mu_{i-1}+x_{i-1}\right)}{\Sigma^{-1}+\Lambda_{i-1}^{-1}}, \\
\Lambda_{i} & =\frac{\Sigma \Lambda_{i-1}}{\Sigma+\Lambda_{i-1}}
\end{aligned}
$$




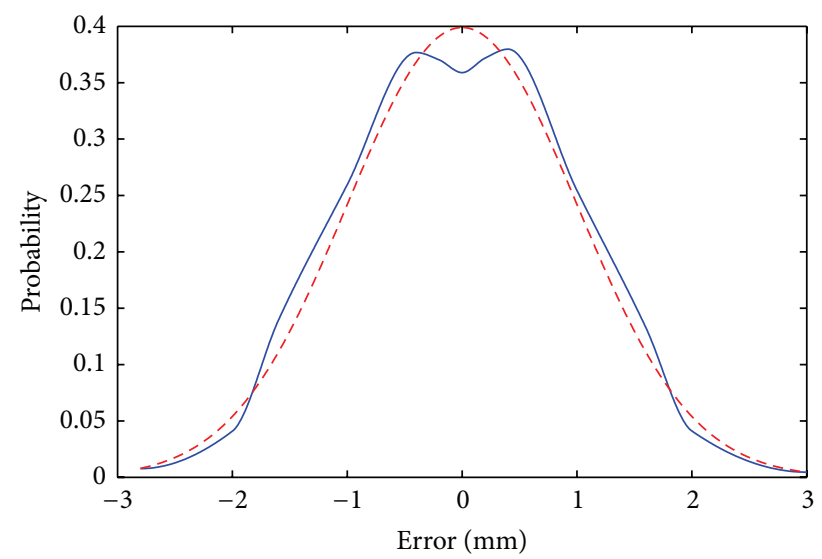

— Predefined noise distribution

- - - Noise approximate distribution

FIgURe 11: Approximate distribution curve of noise.

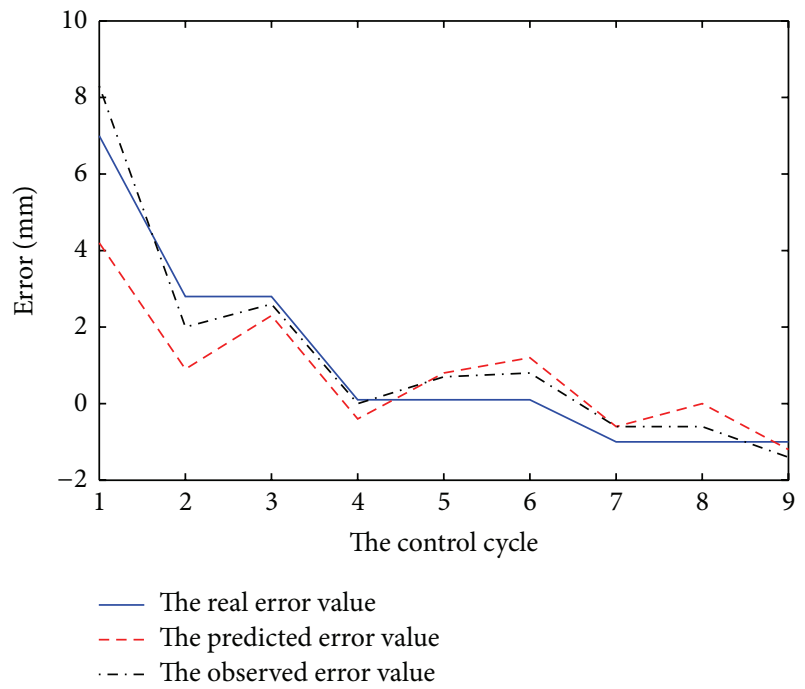

FIGURE 12: Error distribution curve of Bayesian estimation method for a single simulation.

Posterior probability distribution function of $\theta_{i}$ can be described as

$$
f\left(\theta_{i} \mid y_{i}\right)=\frac{1}{\sqrt{2 \pi \Lambda_{i}}} \exp \left\{-\left[\frac{\left(\theta_{i}-\mu_{i}\right)^{2}}{2 \Lambda_{i}}\right]\right\} .
$$

The operational reliability of space manipulator is calculated by the following formula:

$$
\begin{gathered}
P\left\{\theta_{i 1} \leq \theta_{i} \leq \theta_{i 2}\right\}=\int_{\theta_{i 1}}^{\theta_{i 2}} f\left(\theta_{i} \mid y_{i}\right) d \theta_{i} \\
=\int_{\theta_{i 1}}^{\theta_{i 2}} \frac{1}{\sqrt{2 \pi \Lambda_{i}}} \exp \left\{-\left[\frac{\left(\theta_{i}-\mu_{i}\right)^{2}}{2 \Lambda_{i}}\right]\right\} d \theta_{i} .
\end{gathered}
$$

The single and multiple simulation results based on the above method are as shown in Figures 12, 13, and 14.

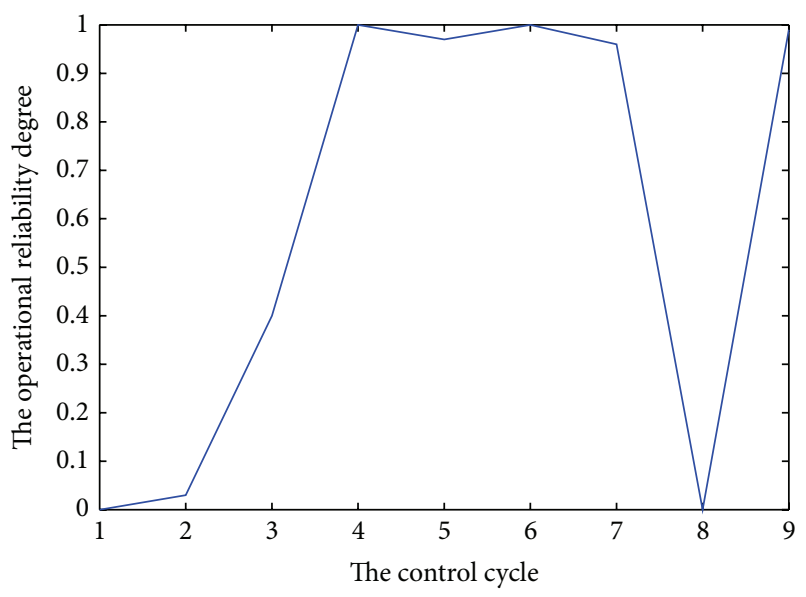

FIGURE 13: Reliability curve of Bayesian estimation method for a single simulation.

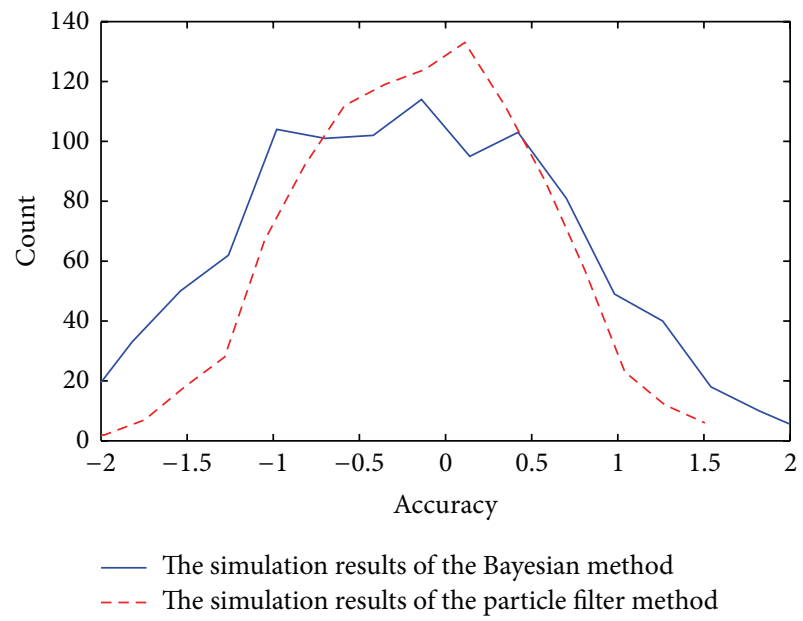

FIGURE 14: Precision distribution curve of Bayesian estimation method for multiple simulation.

As can be seen from the single simulation result in Figure 12, the real value of the terminal error for space manipulator decreases steadily with no fluctuation during the control process and predicted value of error gradually converges to the real error but converges near $-1 \mathrm{~mm}$ with a certain error contrast with the ideal case converging near $0 \mathrm{~mm}$. In addition, it can be seen in Figure 13 that although the degree of the operational reliability estimated by Bayesian method gradually improves, there is a larger fluctuation in the process from the curve compared with the results using the proposed method. Meanwhile, the degree of the operational reliability estimated may be inaccurate due to the approximate distribution of observed noise.

The successful rate of task obtained by Bayesian estimation method is $66 \%$ and the total loss of task is 83531 for 1000 times in Figure 14, which are both inferior compared with those of particle filter algorithm. Therefore, under the described basic conditions, the method proposed in this paper has a more superior performance than that of Bayesian 
estimation method, which can improve operational reliability of manipulator more effectively.

\section{Conclusion}

In this paper, the control model and method based on the degree of the operational reliability for space manipulator are presented and studied, and the following work is mainly completed. First, the control system model of space manipulator based on the degree of the operational reliability is established. Second, the real time control methods for space manipulator based on the degree of the operational reliability are studied, and the estimated distribution of the terminal position error under any observed noise distribution and online calculation of the operational reliability degree are realized using the particle filter algorithm. Then the control performance model is established to realize the real time solution to the adjustment amount of the terminal position. Finally, a control simulation system is established to carry out a large number of simulation verifications, which proves that operational reliability of manipulator and successful rate of task can be improved more effectively by the proposed control method compared with other ones.

\section{Conflict of Interests}

The authors declare that there is no conflict of interests regarding the publication of this paper.

\section{Acknowledgments}

The authors would like to thank their colleagues from the Robotics Research Group for helpful discussions and comments on this paper. Meanwhile, the authors also would like to thank Professor J. William Goodwine at the University of Notre Dame for his valuable and helpful suggestions that led to significant improvements of this paper. This work is supported in part by Key Project of Chinese National Programs for Fundamental Research and Development (973 Program) (no. 2013CB733000) and National Natural Science Foundation of China (no. 61573066).

\section{References}

[1] W. K. Chung, H. Qian, T. L. Lam, and Y. Xu, "Novel design of gaits on space station for dynamic disturbance minimization," IEEE/ASME Transactions on Mechatronics, vol. 19, no. 4, pp. 1392-1403, 2014.

[2] G. Arantes Jr., L. S. Martins-Filho, and A. C. Santana, "Optimal on-off attitude control for the Brazilian Multimission platform satellite," Mathematical Problems in Engineering, vol. 2009, Article ID 750945, 17 pages, 2009.

[3] F. Flacco, A. De Luca, and O. Khatib, "Control of redundant robots under hard joint constraints: saturation in the null space," IEEE Transactions on Robotics, vol. 31, no. 3, pp. 637-654, 2015.

[4] S. Abiko and K. Yoshida, "Adaptive reaction control for space robotic applications with dynamic model uncertainty," Advanced Robotics, vol. 24, no. 8-9, pp. 1099-1126, 2010.
[5] C. Kaiser, P. Rank, and R. Krenn, "Simulation of the docking phase for the SMART-OLEV satellite servicing mission," in Proceedings of the International Symposium on Artificial Intelligence, Robotics and Automation in Space, pp. 26-29, Hollywood, Calif, USA, February 2008.

[6] M. D. Carpenter and M. A. Peck, "Reducing base reactions with gyroscopic actuation of space-robotic systems," IEEE Transactions on Robotics, vol. 25, no. 6, pp. 1262-1270, 2009.

[7] S. A. A. Moosavian, R. Rastegari, and E. Papadopoulos, "Multiple impedance control for space free-flying robots," Journal of Guidance, Control, and Dynamics, vol. 28, no. 5, pp. 939-947, 2005.

[8] E. Zio, "Reliability engineering: old problems and new challenges," Reliability Engineering and System Safety, vol. 94, no. 2, pp. 125-141, 2009.

[9] G. J. Savage and Y. Kap Son, “The set-theory method for systems reliability of structures with degrading components," Reliability Engineering and System Safety, vol. 96, no. 1, pp. 108-116, 2011.

[10] W. R. Brown and A. G. Ulsoy, "A passive-assist design approach for improved reliability and efficiency of robot arms," in Proceedings of the IEEE International Conference on Robotics and Automation (ICRA '11), pp. 4927-4934, Shanghai, China, May 2011.

[11] E. Tian, D. Yue, and C. Peng, "Reliable control for networked control systems with probabilistic sensors and actuators faults," IET Control Theory and Applications, vol. 4, no. 8, pp. 1478-1488, 2010.

[12] A. Charki, A. Demri, F. Guerin, and D. Bigaud, "Mechatronic system reliability evaluation using Petri networks and PHI2 method," in Reliability, Risk and Safety: Theory and Applications, G. Soares, Ed., chapter 221, pp. 1587-1601, CRC Press, 2010.

[13] E. Acar, "Reliability-based design of representative wing and tail system together with structural tests," Journal of Aircraft, vol. 48, no. 6, pp. 2130-2144, 2011.

[14] J. M. Mínguez and J. Vogwell, "Fatigue life of an aerospace aluminium alloy subjected to cold expansion and a cyclic temperature regime," Engineering Failure Analysis, vol. 13, no. 6, pp. 997-1004, 2006.

[15] W. Peng, J. Zhang, and L. Kan, "The reliability analysis of the satellite's driving system based on the IABCBLT method," in Safety and Reliability: Methodology and Applications: Proceedings of the European Safety and Reliability Conference, pp. 143150, CRC Press, 2015.

[16] A. Falcoz, D. Henry, and A. Zolghadri, "Robust fault diagnosis for atmospheric reentry vehicles: a case study," IEEE Transactions on Systems, Man, and Cybernetics Part A: Systems and Humans, vol. 40, no. 5, pp. 886-899, 2010.

[17] J. Gao, X. Gao, W. Zhu, J. Zhu, and B. Wei, "Fault-tolerant and high reliability space robot design and research," in Proceedings of the International Joint Conference on Neural Networks (IJCNN '08), pp. 2413-2417, IEEE, Hong Kong, June 2008.

[18] D. Codetta-Raiteri and L. Portinale, "Dynamic bayesian networks for fault detection, identification, and recovery in autonomous spacecraft," IEEE Transactions on Systems, Man, and Cybernetics: Systems, vol. 45, no. 1, pp. 13-24, 2015.

[19] Y. Kuwata, J. Teo, S. Karaman, G. Fiore, E. Frazzoli, and J. P. How, "Real-time motion planning with applications to autonomous urban driving," IEEE Transactions on Control Systems Technology, vol. 17, no. 5, pp. 1105-1118, 2009. 
[20] W.-K. Yoon, T. Goshozono, H. Kawabe et al., "Model-based space robot teleoperation of ETS-VII manipulator," IEEE Transactions on Robotics and Automation, vol. 20, no. 3, pp. 602-612, 2004.

[21] K. Yoshida, "Engineering test satellite VII flight experiments for space robot dynamics and control: theories on laboratory test beds ten years ago, now in orbit," International Journal of Robotics Research, vol. 22, no. 5, pp. 321-335, 2003.

[22] H. Chaoui, P. Sicard, and W. Gueaieb, "ANN-based adaptive control of robotic manipulators with friction and joint elasticity," IEEE Transactions on Industrial Electronics, vol. 56, no. 8, pp. 3174-3187, 2009.

[23] D. Hsu, R. Kindel, J.-C. Latombe, and S. Rock, "Randomized kinodynamic motion planning with moving obstacles," The International Journal of Robotics Research, vol. 21, no. 3, pp. 233255, 2002.

[24] M. Stilman, "Global manipulation planning in robot joint space with task constraints," IEEE Transactions on Robotics, vol. 26, no. 3, pp. 576-584, 2010.

[25] H. Sadeghian, L. Villani, M. Keshmiri, and B. Siciliano, "Taskspace control of robot manipulators with null-space compliance," IEEE Transactions on Robotics, vol. 30, no. 2, pp. 493-506, 2014.

[26] M. Kobilarov, "Nonlinear trajectory control of multi-body aerial manipulators," Journal of Intelligent \& Robotic Systems, vol. 73, no. 1-4, pp. 679-692, 2014.

[27] J. Rosell, R. Suárez, C. Rosales, and A. Pérez, "Autonomous motion planning of a hand-arm robotic system based on captured human-like hand postures," Autonomous Robots, vol. 31, no. 1, pp. 87-102, 2011.

[28] Y. Wang, X. Gao, H. Sun, Q. Jia, W. Zhao, and M. Du, "Research on control method based on real-time operational reliability evaluation for space manipulator," Advances In Mechanical Engineering, vol. 6, Article ID 179293, 2014.

[29] M. Sanjeev Arulampalam, S. Maskell, N. Gordon, and T. Clapp, "A tutorial on particle filters for online nonlinear/non-Gaussian Bayesian tracking," IEEE Transactions on Signal Processing, vol. 50, no. 2, pp. 174-188, 2002.

[30] Z. Lian and E. del Castillo, "Setup adjustment under unknown process parameters and fixed adjustment cost," Journal of Statistical Planning and Inference, vol. 136, no. 3, pp. 1039-1060, 2006.

[31] O. Cappe, S. J. Godsill, and E. Moulines, "An overview of existing methods and recent advances in sequential Monte Carlo," Proceedings of the IEEE, vol. 95, no. 5, pp. 899-924, 2007. 


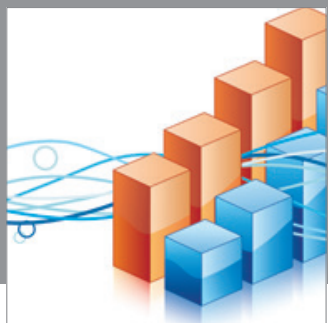

Advances in

Operations Research

mansans

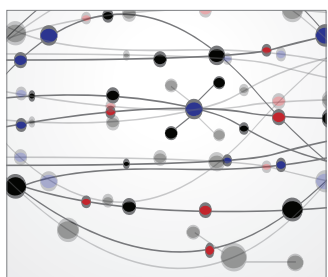

The Scientific World Journal
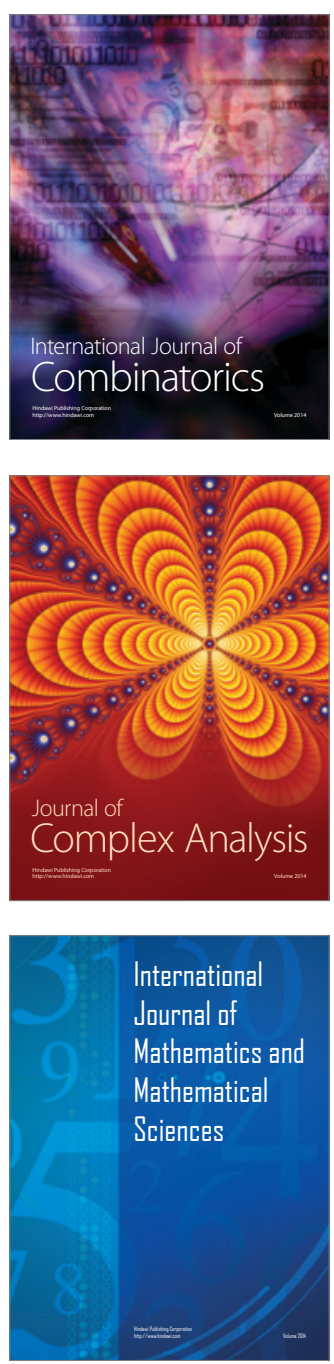
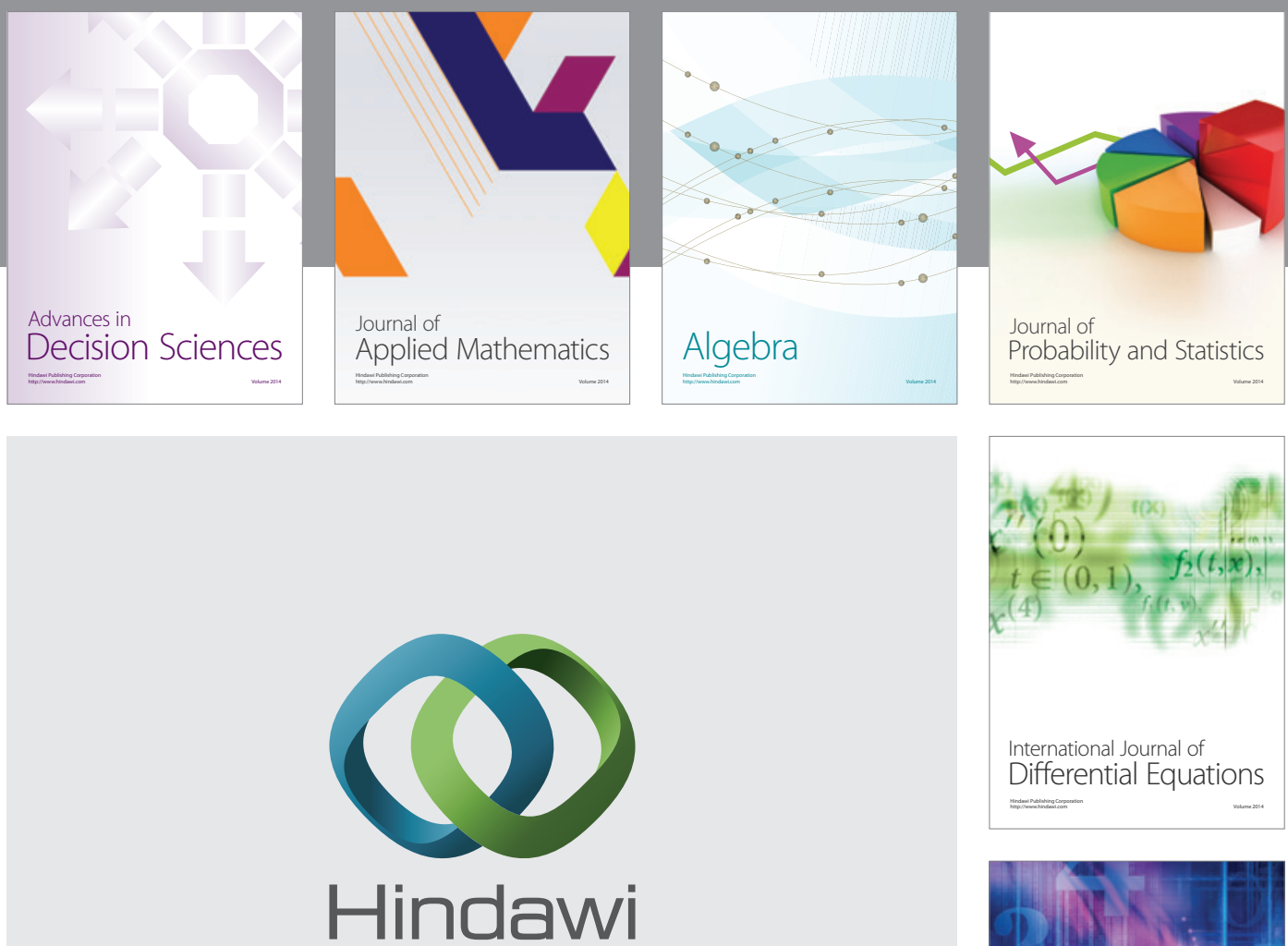

Submit your manuscripts at http://www.hindawi.com
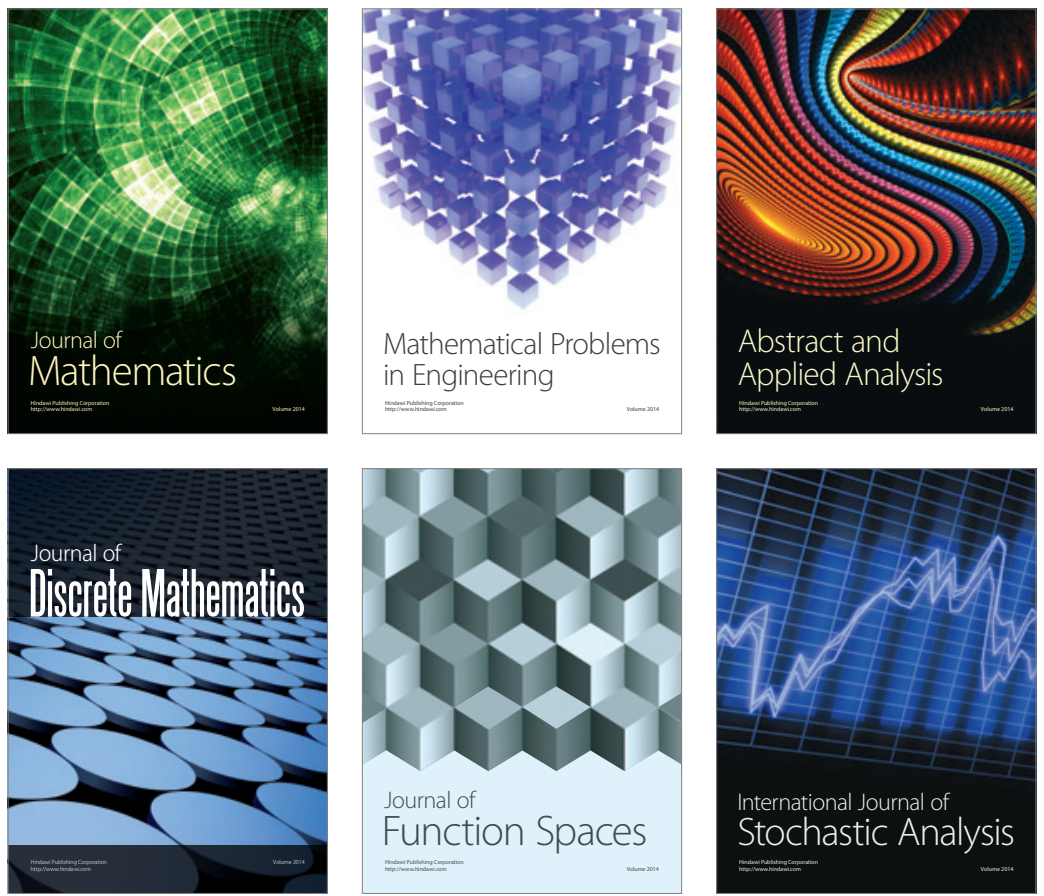

Journal of

Function Spaces

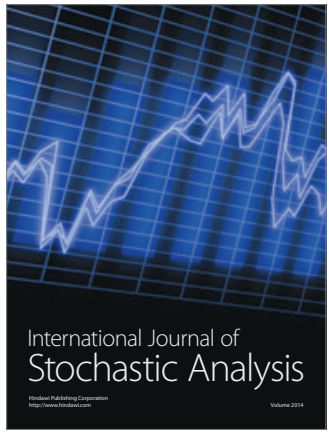

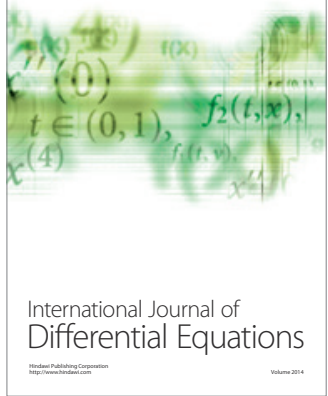
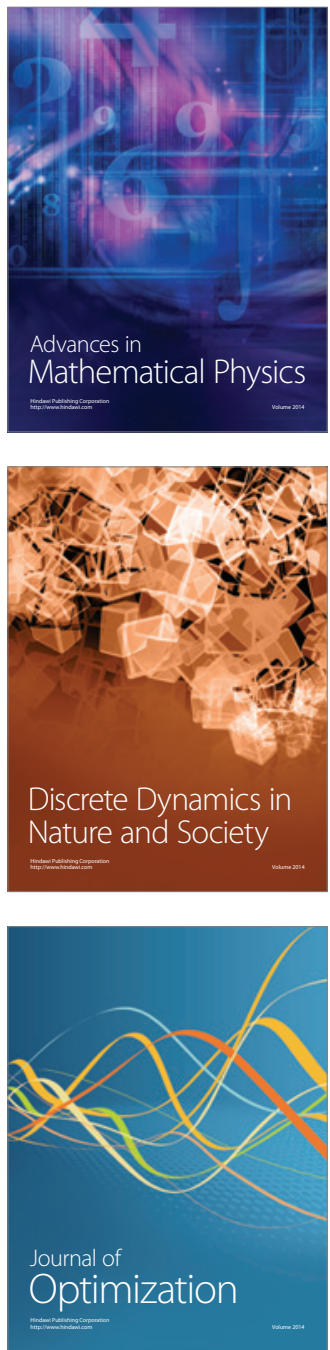